\title{
A Case of Cancer of the Ampulla of Vater Accompanied by Malrotation
}

\author{
RYUICHI KAWAHARA, HIROYUKI HORIUCHI, HIDEFUMI NOGITA, MASANORI AKASHI, \\ KAZUHIRO MIKAGI, MUNEHIRO YOSHITOMI, GEN AKASU, YUHEI KITASATO, \\ YUSUKE KAWASHIMA, HIROTO ISHIKAWA, TOHRU HISAKA, \\ HISAFUMI KINOSHITA AND HIROYUKI TANAKA
}

Department of Surgery, Kurume University School of Medicine, Kurume 830-0011, Japan

Received 11 September 2012, accepted 13 March 2013

J-STAGE advance publication 7 August 2013

\begin{abstract}
Summary: Intestinal malrotation is caused by a developmental anomaly of the embryonic intestine. Most cases develop in neonates, and development in adulthood is rare and difficult to diagnose before surgery.

Pancreaticoduodenectomy was performed for cancer of the ampulla of Vater accompanied by incomplete fixation in a 63-year-old male patient. A branch of the superior mesenteric artery was present on the resection line and was deemed likely to cause circulatory disorder in the small intestine, and the duodenum and jejunum were covered with a membranous structure making dissection, anatomical identification, and jejunectomy difficult. Herein, we report the case with a review of the literature.
\end{abstract}

Key words incomplete fixation, paraduodenal hernia, malrotation, intestinal volvulus, pancreaticoduodenectomy

\section{INTRODUCTION}

Intestinal malrotation is caused by a developmental anomaly of the embryonic intestine. Most cases develop in neonates, and development in adulthood is rare and difficult to diagnose before surgery.

We encountered a patient in whom we suspected intestinal malrotation during pancreaticoduodenectomy, and made a diagnosed is of incomplete fixation based on re-examination by imaging after surgery. Herein, we report the case with a review of the literature.

\section{CASE REPORT}

The patient was a 63-year-old male who visited a physician for jaundice. Cancer of the ampulla of Vater was diagnosed based on thorough examination, and the patient was referred to our hospital. There was no past medical history of tumors or surgery.

Blood test findings: On admission, alanine aminotransferase (ALT) and $\gamma$-glutamyl transpeptidase
$(\gamma$-GTP) were high (39 and $153 \mathrm{U} / \mathrm{L}$, respectively), and total bilirubin ( $\mathrm{T}$ Bil) was normal $(0.69 \mathrm{mg} / \mathrm{dl})$. Carcinoembryonic antigen was $1.1 \mathrm{ng} / \mathrm{ml}$ (normal range $0-0.5 \mathrm{ng} / \mathrm{ml}$ ), and carcinoma antigen $19-9$ was $23.3 \mathrm{U} / \mathrm{ml}$ (normal range $0-37 \mathrm{ng} / \mathrm{ml}$ ).

On plain X-ray radiography on admission, no ileus was noted, and the colon gas location was normal. On abdominal ultrasonography, the superior mesenteric artery (SMA) and vein (SMV) could not be closely observed. On abdominal computed tomography (CT), swelling to a maximum size of $20 \mathrm{~mm}$ was present in the papillary region, and the common and intrahepatic bile ducts were dilated. The SMA was located on the left side of the SMV, showing no SMV rotation sign (Fig. 1A). A whirlpool sign showing whirling SMV, mesentery, and intestine around the SMA was observed (Fig. 1B). On three-dimensional CT reconstruction imaging, the small intestine showed rightward deviation, but the colon position appeared normal. The whirlpool sign was also observed (Figs. 2A and B).

In surgery, Kocher mobilization was applied and

Address Correspondence to: Department of Surgery, Kurume University School of Medicine 67 Asahi-machi, Kurume-shi, Fukuoka-ken 830-0011, Japan. Tel: +81-942-31-7567 Fax: +81-942-35-8967 E-mail: ryukawa@med.kurume-u.ac.jp

Abbreviations: ALT, alanine aminotransferase; CT, computed tomography; SMA, superior mesenteric artery; SMV, superior mesenteric vein; T Bil, total bilirubin; $\gamma$-GTP, $\gamma$-glutamyl transpeptidase. 

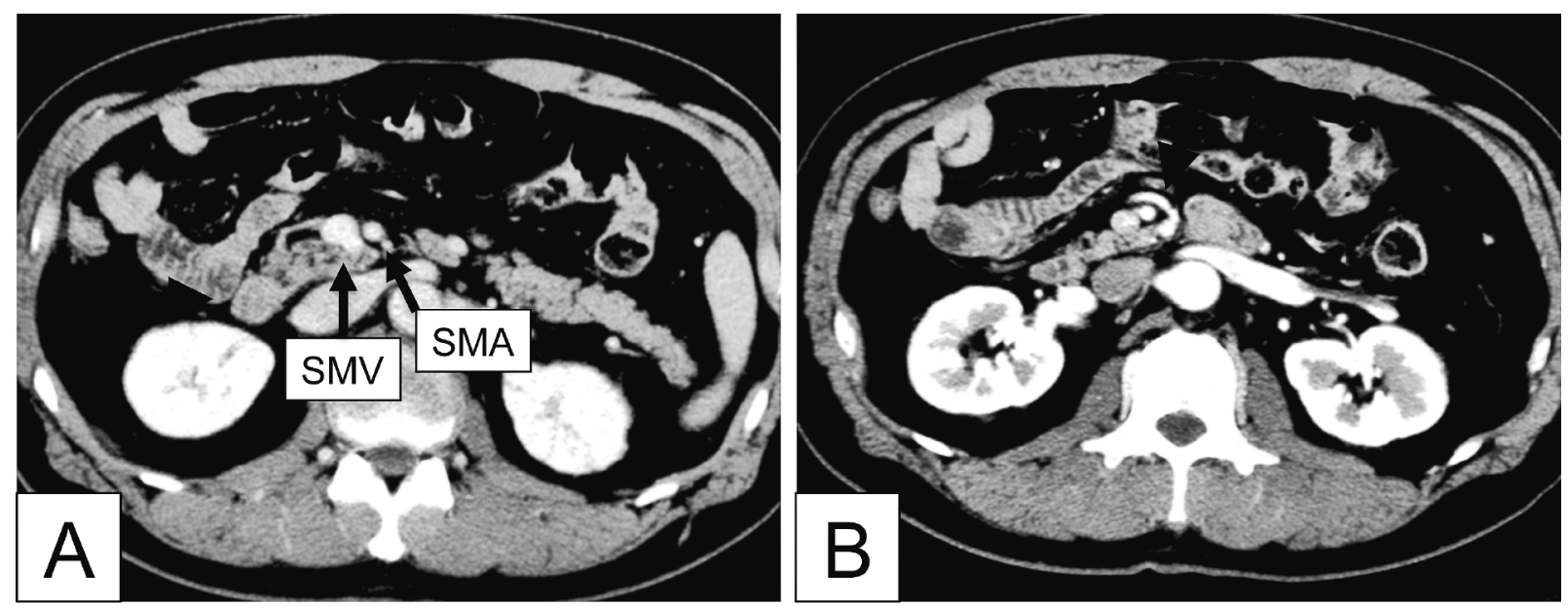

Fig. 1. CT findings.

Swelling to maximum size $20 \mathrm{~mm}$ was present in the papillary region (white arrow).

A: The superior mesenteric artery (SMA) was located on the left side of the superior mesenteric vein (SMV), showing no SMV rotation sign (black arrow).

B: Wirlpool sign showing SMV, mesentery, and intestine around the SMA was observed (white arrow).
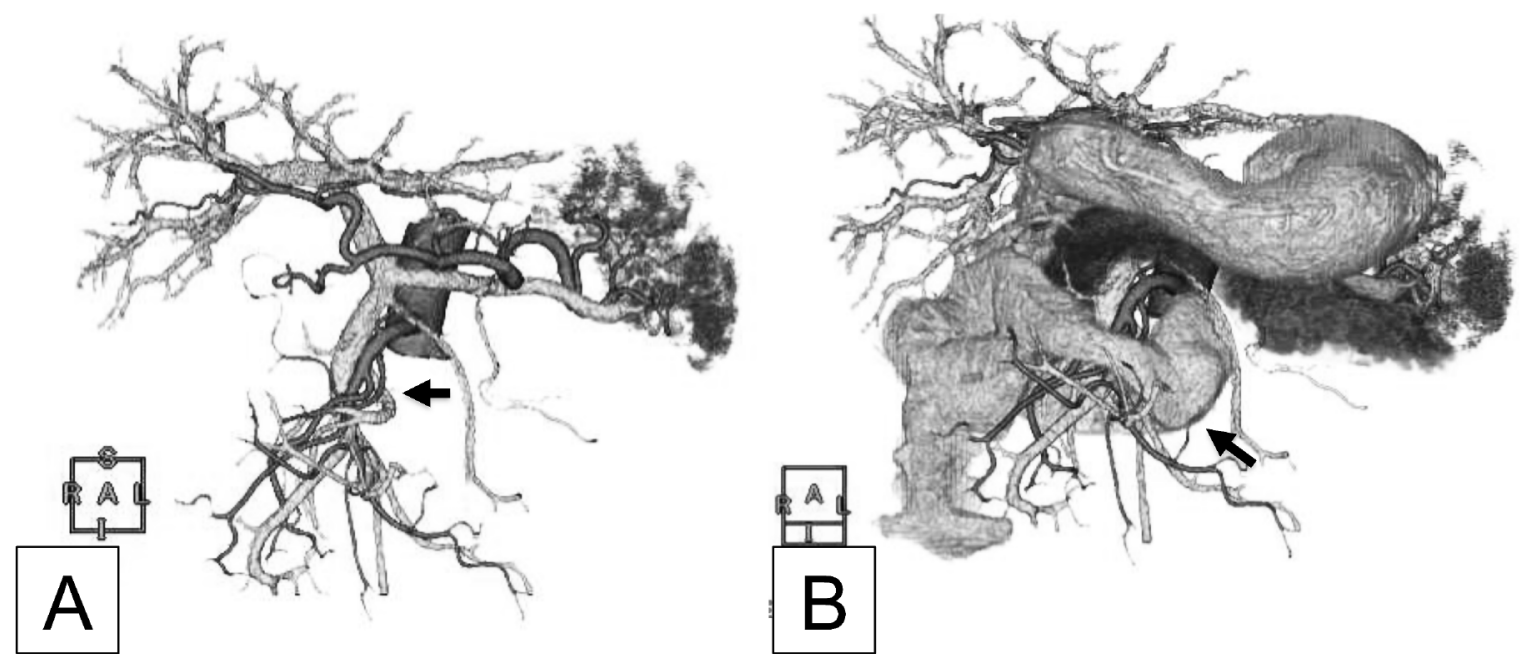

Fig. 2. 3D reconstruction imaging of CT.

A: Whirlpool sign showing whirling SMV, mesentery, and sign was also observed (black arrow).

B: The small intestine was deviated rightward. The whirlpool sign was also observed (black arrow).

the duodenum was mobilized, but the duodenum and jejunum were covered with a membranous structure and dissection was difficult. Thus, we decided to start dissection from the Treitz' ligament side of the jejunum (Fig. 3), but the Treitz' ligament was absent, and the jejunum had rotated rightward around the SMA. Division of the minor vascular and retroperitoneal attachments was complicated by anomalous vascular orientation, but pancreaticoduodenectomy could be performed (Fig. 4). Based on the intestinal volvulus, no change in ischemic bowel obstruction or strangulation was observed.

The pathological diagnosis was tubular adenocarcinoma, well-differentiated, $2.2 \times 2.0 \mathrm{~cm}$, pat Adcb, depth: $\mathrm{m}$, medullary type, ly0, v0, pn0, pPanc0, pDu0, Pn0, PeMo, n;(-), pT1, pNO, pM(-) fStage I.

\section{DISCUSSION}

The intestine (midgut) perfused by the SMA develops outside the abdominal cavity, returns to the abdominal cavity while rotating 270 degrees around the 


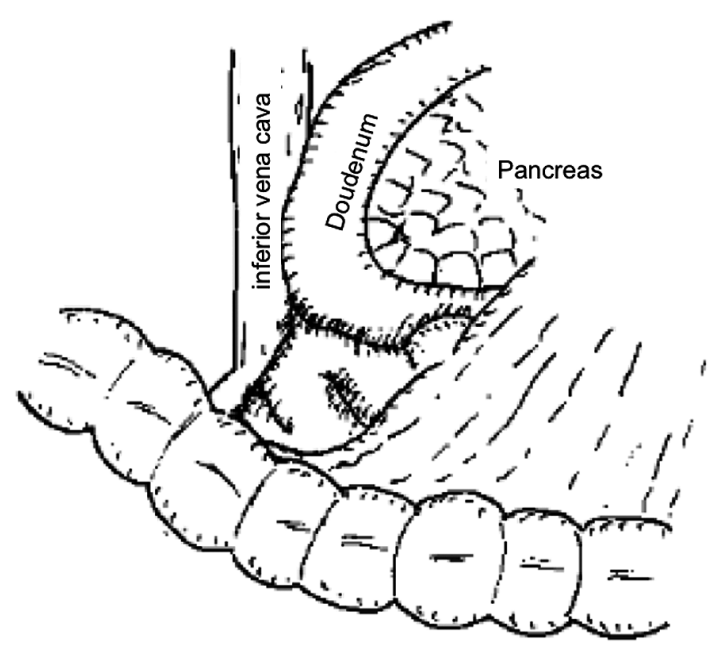

Fig. 3. Operative findings 1.

Kocher mobilization was applied and the duodenum was mobilized, but the duodenum and jejunum were covered with a membranous structure and dissection was difficult.

TABLE 1.

Classification of the rotation
1) nonrotation
2) incomplete rotation
(1) incomplete rotation both limbs
(2) incomplete rotation of the doudenojekunal limb
(3) nonrotation of the cecocolic limb
(4) reverse rotation
3) incomplete fixation
(1) mesocolic hernia
(2) mobile cecum

SMA as an axis, and becomes fixed to the retroperitoneum at about 10 weeks of gestation. Malrotation and abnormal fixation to the peritoneum/retroperitoneum in this step are collectively termed intestinal malrotation. Intestinal malrotation is described by classifying rotation into 0,90 , and 180 degrees, inverse rotation, and others, but no consistent viewpoint has been reached [1-3], and terms vary, for example secession of rotation at 0 or 90 degrees may be termed nonrotation, secession at 180 degrees may be termed malrotation or incomplete rotation, inverse rotation may be termed malrotation or reversed rotation, and others may be termed paraduodenal hernia. Nishijima et al. [4] roughly divided these case into 3 types classified by Bill into

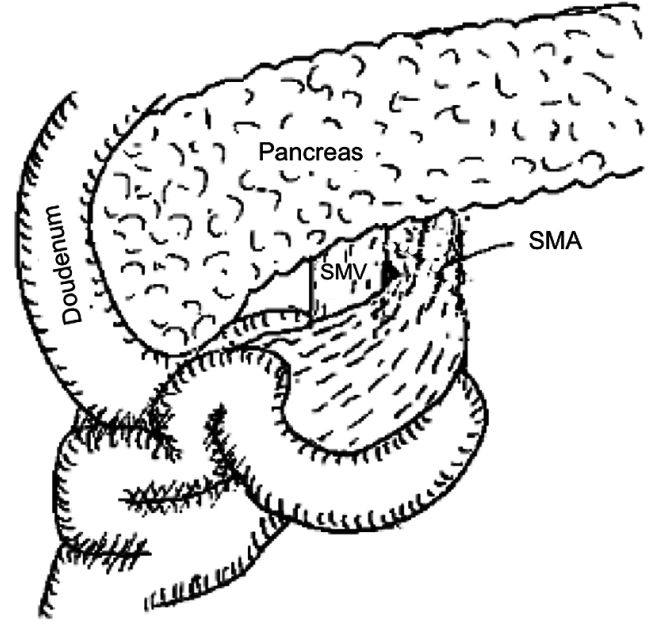

Fig. 4. Operative findings 2.

The Treitz's ligament was absent, and the jejunum was rotated rightward around the SMA. Dissection of the mesentery and jejunum was difficult.

the following categories in consideration of various mixed and transition types: 1) Nonrotation type, 2) incomplete rotation type, and 3 ) incomplete fixation, and subdivided 2) (incomplete rotation) into incomplete rotation of both limbs, incomplete rotation of the duodenojejunal limbs, nonrotation of the cecocolic limbs, and reverse rotation, and 3) (incomplete fixation) into mesocolic hernia and mobile cecum (Table. 1). The incidences of nonrotation and incomplete rotation of both limbs are high, and various studies have been reported [5-8].

When the association between pancreaticoduodenectomy and intestinal malrotation was searched for in Pubmed with regard to malrotation, incomplete rotation, reverse rotation, and pancreaticoduodenectomy, 4 reported cases were found [9-12]. No report of an incomplete fixation type of intestinal malrotation, as seen in the present case, was found. In this type, a hernial sac is formed, and may lead to the development of an internal hernia, causing intestinal compression, obstruction, and strangulation. The present patient had no symptoms of a hernia.

An understanding of vascular variants is critical when performing a pancreaticoduodenectomy in malrotated patients. Failure to appreciate the anomalous location of the superior mesenteric artery to the left of the superior mesenteric vein can result in devascularization of the short segmental vessels feeding the small bowel. As each of these small vessels is essentially an 
end artery, their ligation can lead to small bowel death with catastrophic outcomes. This report stated that the presence of the SMA on the resection line is a characteristic of this type, and resection may cause circulatory disorder of the small intestine. In our patient, the Treitz' ligament was absent, and the mesentery was present twining around the ventral side of the SMV. Careful attention should be paid to avoid injury to the mesentery during dissection of the SMV and division of the jejunum. Moreover, in our case when we applied Kocher mobilization we found that the duodenum and jejunum were covered with a membranous structure made dissection and anatomical identification difficult. The membranous structure was widely present, but no Ladd's ligament was noted $[6,13,14]$. Division of the jejunum was also difficult because the intestine was present in a lump.

For preoperative diagnosis, a whirl-like pattern or whirlpool sign representing the intestine twining around the SMA or SMV rotation sign with the SMA located on the left side of the SMV on abdominal ultrasonography and CT are reportedly useful [15-18]. In this patient, the lesion was located lower than the observational range of abdominal ultrasonography, and no diagnosis could be made. On CT, the above sign was noted, but we could not diagnose the syndrome before surgery. Diagnosis may have been possible if the image had been read in consideration of intestinal malrotation.

In conclusion, we reported a rare case in whom dissection anatomical identification, and jejunectomy were difficult because a branch of the superior mesenteric artery was present on the resection line during surgery such that injury to the SMA was likely to cause circulatory disorder of the small intestine, and the duodenum and jejunum were covered with a membranous structure. The possibility of malrotation should be taken into consideration when performing pancreatoduodenectomy.

\section{REFERENCES}

1. Alexander H Bill. Malrotation of the intestine, ed. Ravitch MM. Pediatric Surgery Yearbook Medical Publishers, Chicago 1979; 11.

2. Chaffin L and Snyder WH. Malrotation of the intestine. Surg Clin North Am 1956; 36:1479-1494.

3. Wang CA and Welch CE. Anomalies of Intestinal Rotation in Adolescents and Adults. Surgery 1963; 54:839-855.

4. Nishijima E. Malrotation as a Spectrum of Anomalous Bowel Rotation and Fixation with a Wide Range of Clinical Findings. Japanese Journal of pediatric surgery. 2005; 37(7):749-754.

5. Ren PT and $\mathrm{Lu} \mathrm{BC}$. Intestinal malrotation associated with colon cancer in an adult: report of a case. Surg Today 2009; 39(7):624-627.

6. Yamashita H, Kato H, Uyama S, Kanata T, Nishizawa F et al. Laparoscopic repair of intestinal malrotation complicated by midgut volvulus. Surg Endosc 1999; 13(11):11601162.

7. Fu T, Tong WD, He YJ, Wen YY, Luo DL et al. Surgical management of intestinal malrotation in adults. World $\mathrm{J}$ Surg 2007; 31(9):1797-1803; discussion 1804-1805.

8. Kanazawa T, Kasugai K, Miyata M, Miyashita M, Mizuno $\mathrm{M}$ et al. Midgut malrotation in adulthood. Intern Med 2000; 39(8):626-631.

9. Hayashi T, Takano S, Kimura F, Shimizu H, Yoshidome H et al. A case of cholangiocarcinoma with hepatomesenteric trunk and intestinal malrotation treated with pancreaticoduodenectomy. Gan To Kagaku Ryoho 2010; 37(12):27232725.

10. Plackett TP, Takamori $\mathrm{R}$, and Izawa $\mathrm{M}$. Pancreaticoduodenectomy in the setting of intestinal malrotation. Hawaii Med J 2011; 70(11):237-238.

11. Jagannath P, Albuquerque K, de Souza LJ, Mohandas KM, and Shantiswaroop V. Reversed rotation of midgut which caused problems during Whipple's procedure. Eur J Surg 1995; 161(10):765-766.

12. Daniels BT, Clader DN, Luter PW, Coppinger WR, and Good GT. Pancreatico-duodenectomy in incomplete bowel rotation, anatomical peculiarities. Rocky Mt Med J 1957; 54(5):445-447.

13. Stanfill AB, Pearl RH, Kalvakuri K, Wallace LJ, and Vegunta RK. Laparoscopic Ladd's procedure: treatment of choice for midgut malrotation in infants and children. $\mathrm{J}$ Laparoendosc Adv Surg Tech A 2010; 20(4):369-372.

14. Raphaeli T, Parimi C, Mattix K, and Javid PJ. Acute colonic obstruction from Ladd bands: a unique complication from intestinal malrotation. J Pediatr Surg 2010; 45(3):630-631.

15. Pracros JP, Sann L, Genin G, Tran-Minh VA, Morin de Finfe $\mathrm{CH}$ et al. Ultrasound diagnosis of midgut volvulus: the "whirlpool" sign. Pediatr Radiol 1992; 22(1):18-20.

16. Green P, Swischuk LE, and Hernandez JA. Delayed presentation of malrotation and midgut volvulus: imaging findings. Emerg Radiol 2007; 14(6):379-382.

17. Zissin R, Rathaus V, Oscadchy A, Kots E, Gayer G et al. Intestinal malrotation as an incidental finding on $\mathrm{CT}$ in adults. Abdom Imaging 1999; 24(6):550-555.

18. Clark P and Ruess L. Counterclockwise barber-pole sign on CT: SMA/SMV variance without midgut malrotation. Pediatr Radiol 2005; 35(11):1125-1127. 